types of radiation bend space-time in such a way that the region from which the Hawking radiation is generated eventually gets pushed back behind the event horizon. Once it has been relocated, the radiation can no longer escape and the black hole plateaus at a constant mass.

The analysis is more of an essay than a fullblown study, says string theorist Samir Mathur of Ohio State University in Columbus, because Ellis does not perform a thorough calculation for the bending effect of the radiation. Other physicists say that Ellis is probably incorrect. Nonetheless, Balasubramanian says, the paper is an example of physicists' innate dissatisfaction with evaporating black holes. "The stuff radiates in a weird way, information is lost and then we turn intellectual somersaults to try to account for why the information isn't lost," he says.

But black hole remnants do not offer a perfect solution to the problem of information loss, either. To contain all the information originally stored in a large black hole, the tiny remnants would need to have an infinite number of internal states - which would violate quantum theory, says Mathur.

In 1997, Mathur found a potential way around this problem. He and his colleagues used string theory, a way of marrying quantum theory and gravity, to describe all of the possible states of the gravitational field of black holes. They found that these states lay just outside the classical event horizon in a 'fuzzball' of quantum states. If the fuzzball was the black hole's true boundary, then there would be no Hawking radiation emitted from the event horizon, no evaporation and no information loss, he says.

Last year, others proposed a more violent approach to the problem of information loss. They suggested that the particles in the Hawking radiation did not behave randomly but were instead 'entangled' with each other in such a way that they could be messengers from the darkness, conveying information that had been stored in the black hole. But that entanglement had its own consequence: an enormous amount of energy would be unleashed at the event horizon, such that anything falling in would be instantly immolated (see Nature 496, 20-23; 2013).

The ongoing struggle to preserve the precepts of quantum theory without losing information may offer clues about how and where quantum physics begins to significantly modify Einstein's theory of gravity, says Matt Visser, a cosmologist at Victoria University of Wellington. The conventional picture holds that quantum theory makes big corrections to gravity only well inside the event horizon, near the black hole's singularity - the point at which the density of matter becomes infinite. But some physicists think that quantum physics may be blurring the sharp boundary of the event horizon itself. Ellis's work, Visser says, puts a stronger spotlight on such speculations. -

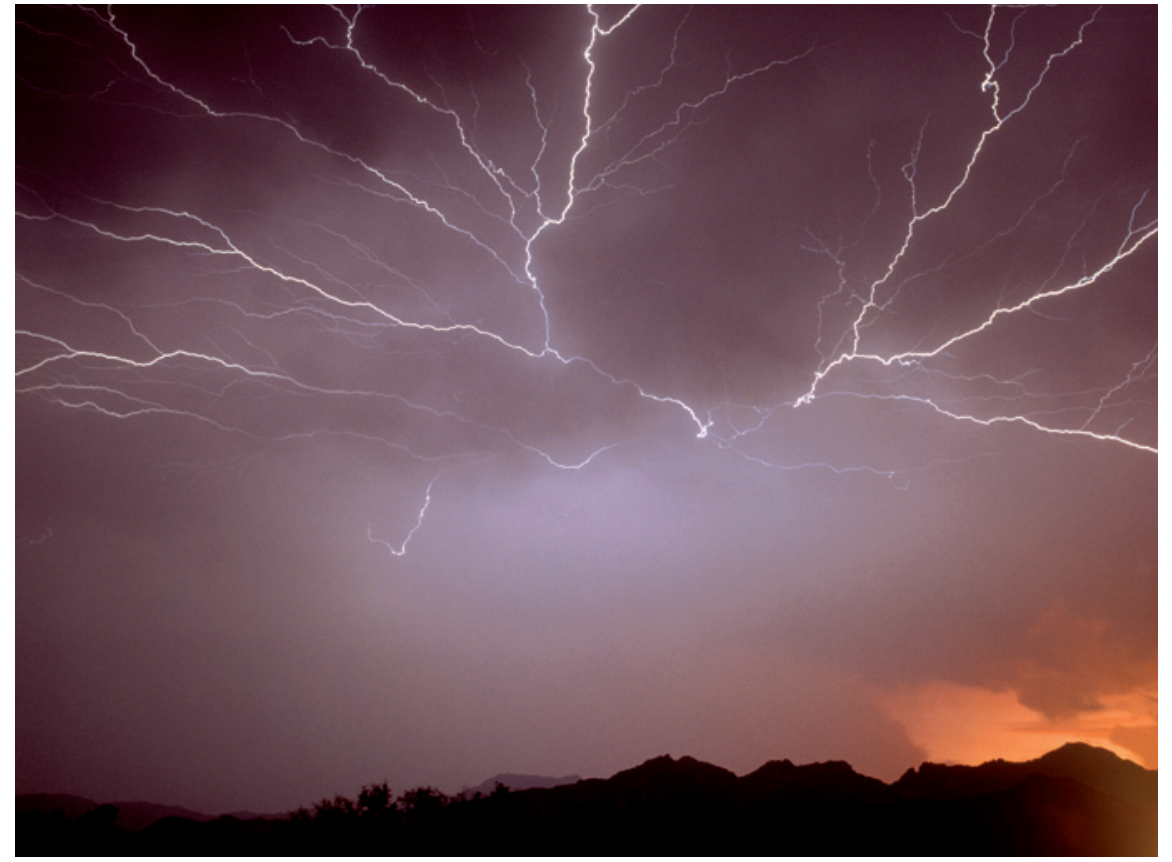

Cloud-to-cloud lightning can be detected with sensors, and used as a proxy for intense storms.

\title{
METEOROLOGY
}

\section{Lightning network tested out in Guinea}

\author{
Project to forecast African storms provides cheap \\ alternative to radar-based weather services.
}

\section{BY JEFF TOLLEFSON}

$\mathrm{M}$ eteorologists watched as afternoon thunderstorms brewed in the mountainous region of central Guinea. By the evening of 22 October, the storms had intensified and were moving west towards the coast of Africa. At 8.20 p.m., the meteorologists received a thunderstorm alert, and for the next 45 minutes the 130,000 residents of the city of Fria were hammered by heavy rain, flash floods and winds of up to 77 kilometres per hour.

What happened that evening was not unusual. Similar storms blow through Fria and Guinea's coastal capital Conakry regularly during the rainy season. Flash flooding is a common problem, and the country is frequently buffeted by tornadoes.

What was unusual was the way the storm was detected. Government meteorologists in Guinea lack the Doppler radar system that is usually used for this, and have struggled to track weather using rudimentary equipment. Europe and the United States provide free satellite data and forecasts, but these are coarse and infrequent. Only in recent months has Guinea turned to a new, simple proxy for storms: flashes of lightning.

Lightning-detection sensors installed atop just 12 mobile-phone towers now allow the country's meteorological service to track storms nationwide as they develop (see 'Lightning fast'). "With this project, we receive virtually real-time data throughout the entire country," says Mamadou Lamine Bah, director of the service.

The project shows how lightning detection could provide a quick and relatively cheap way for poor countries to acquire basic weather services. Earth Networks, based in Germantown, Maryland, spent around US\$1 million to deploy the current network. By comparison, Bah says, even a simple Doppler radar system would cost around \$10 million. And beyond that initial outlay, a lack of expertise in countries such as Guinea makes those systems prohibitively difficult to maintain and operate.

Earth Networks is currently giving Guinea its lightning data for free, but eventually hopes to sell its services there. Already, the company 


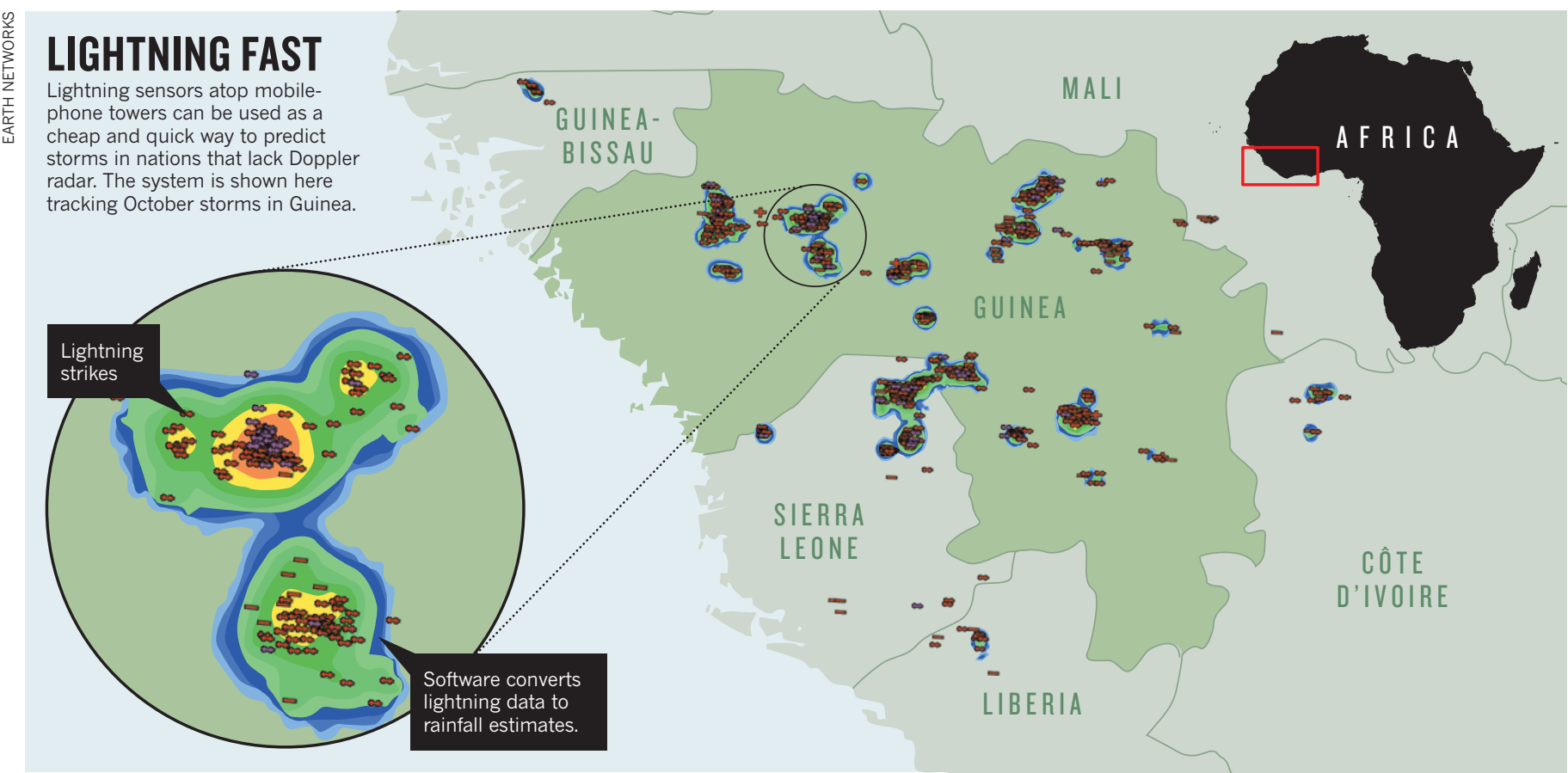

has contracts worth more than $\$ 5$ million with US government bodies, including the National Oceanic and Atmospheric Administration (NOAA) and the Air Force, to provide lightning data from nearly 700 sensors across North America and the Caribbean. It also has smaller customers, such as golf courses and airports. "Our system will work in any part of the world and instantaneously produce results," says Robert Marshall, Earth Networks' president and chief executive. "That's the real breakthrough here."

The technology has its roots in research carried out at the Massachusetts Institute of Technology in Cambridge, and depends on a 26-centimetre-long antenna that registers high-frequency electromagnetic signals from lightning discharges. Earth Networks acquired the technology in 2006, and developed the software necessary to triangulate among multiple sensors to pinpoint individual flashes.

The company has competitors, including Vaisala, based in Vantaa, Finland. But Earth Networks was the first, in 2009, to deploy a system for tracking not just the major lightning bolts that strike the ground but also the more common cloud-to-cloud lightning strikes that are generated as storms intensify.

Earth Networks uses the data to assess storm activity and estimate rainfall, and produces severe-storm alerts 9-16 minutes faster than those generated by NOAA's National Weather Service using Doppler radar.

NOAA is now looking at ways to incorporate lightning data into high-resolution weather models to improve storm tracking. "It's a pretty powerful technology," says Alexander MacDonald, director

of NOAA's Earth Sys-

tem Research Labo-

ratory in Boulder,

Colorado. Such data

\section{“It's a pretty powerful technology."}

will initially come

from Earth Networks' tower antennas, but MacDonald says that the agency will eventually collect lightning data from its next geostationary weather satellite, scheduled for launch in 2016.

But the technology's biggest impact could be in countries with limited access to weather services based on satellites and radar. Earth Networks has more than 50 antennas covering most of Brazil and 50 more covering all of India. The company says that it has contracts worth a total of several million dollars in the two countries. The project in Guinea is intended to showcase the technology in a country that has little scientific capacity and hardly any meteorological infrastructure at all.

John Snow, an atmospheric scientist at the University of Oklahoma in Norman and author of a forthcoming World Bank report on weather services in developing countries, says that the Guinea project is an attractive model. The phone towers, which also have basic weather sensors, do not provide ideal conditions for weather stations, Snow says, but the telecommunications company with which Earth Networks has partnered can provide security, technicians and communications. Although custom-built weather stations might provide more-precise data, using phone towers is cheaper and more reliable, he says. "It is a working solution that certainly moves us in the right direction."

Bah says that his agency must now find ways to disseminate the alerts, perhaps by phone. It currently broadcasts radio forecasts just two or three times a day. It must also find money to eventually pay Earth Networks and to formally incorporate lightning data into its operations. But in just a few months, Guinea has moved from rough forecasts to real-time storm tracking. "What I can say from our evaluation is that this system can be deployed in countries like Guinea," says Bah. .

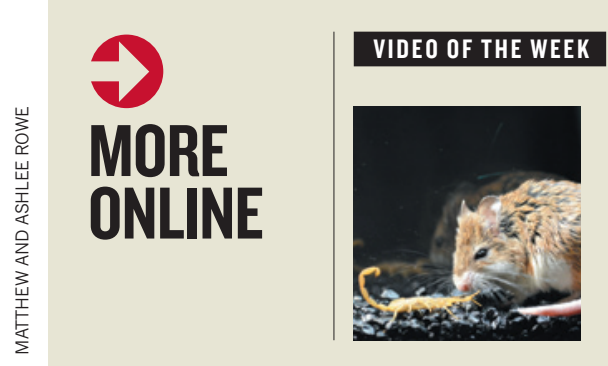

Mouse species' resistance to pain of scorpion venom hints at potential drug target go.nature.com/zqtgim

\section{MORE NEWS}

- PubMed opens post-publication comment platform go.nature.com/dgftwk - Astronomers will train on simulated data for dark-energy survey go nature. com/79ekb5

- Atmospheric patterns predict extreme US weather go.nature.com/fjsuha

\section{NATURE PODCAST}

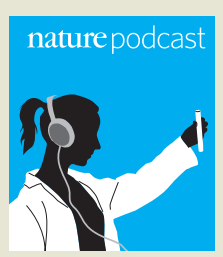

Treating chronic HIV; time travel in fiction; and a book on physics under the Nazi regime nature.com/ nature/podcast 